$(66 \%)$ parents wanted to continue with this form of therapy. They found the technique to be uncomplicated, painless and useful. None of our patients became worse or had side effects.

Conclusion Neuromodulation is a noninvasive method which was found to be useful in improving stool regulation by more than half of our patients with chronic intractable constipation when administered as a complementary method to standard therapy and biofeedback. As this study included a small number of patients it would be desirable to plan a prospective study which will actuate a larger number of patients and long-term monitoring.

\section{FOREIGN BODY INGESTION IN CHILDREN - FIVE YEARS OF EXPERIENCE AFTER THE ADOPTION OF GUIDELINES}

${ }^{1}$ Matea Kovačićx ${ }^{1}{ }^{1}$ Nika Puževski, ${ }^{1,2}$ Mirna Natalija Aničić, ${ }^{1}$ Lana Omerza, ${ }^{1,2}$ Irena SenečićČala, 1,2Duška Tješić-Drinković, ${ }^{1,2}$ Jurica Vuković. ${ }^{1}$ UHC Zagreb, Department of Pediatrics; ${ }^{2}$ University of Zagreb, School of Medicine

10.1136/archdischild-2021-europaediatrics.281

Objective Reevaluation of our experiences in adherence to the established local guidelines in our population of pediatric patients diagnosed with foreign body ingestion.

Methods A retrospective study of patients aged 0-18 years who were admitted to pediatric emergency department of the University Hospital Centre Zagreb between 1.1.2015. and 31.12.2019. due to suspected foreign body ingestion. We grouped them according to their age and localization of the foreign body along the digestive tract. We analyzed how many patients and with what success underwent endoscopy in relation to the applicable guidelines in our Institution.

Results Of the 410 patients with suspected foreign body ingestion, a foreign body was found in 175 patients ( $x=4$ years \pm 9 months), more common in male children (100/175). Most of them $(78 / 175,45 \%)$ were in the age group 3-7 years, followed by $1-3$ year group $(51 / 175,29 \%)$. Foreign body was localized radiologically in 165 (94\%), by endoscopy in $8(5 \%)$ patients, while in the two patients localization hasn't been determined, and there was also one spontaneous foreign body expulsion. Most common foreign bodies were coins in 61 children (35\%), followed by another metal object in 51 (29\%), button battery in $35(20 \%)$ and plastic object in 7 (4\%) patients. In 11 children (6\%) it was food bolus impaction, and 10 of them swallowed other objects. Most foreign bodies were localized in the stomach (95 patients, 54\%), followed by the small intestine (38 patients, 22\%), the esophagus (27 patients, 15\%) and the colon (9 patients, 5\%). Two toothpicks were found in piriform sinus and tonsils. Endoscopy was performed in a third of patients $(59 / 175 ; 34 \%)$, and it was successful (resulting in foreign body extraction) in 48 of them $(81 \%)$. In $25 / 27$ of patients with foreign body localized in the esophagus endoscopy was performed, while the two asymptomatic patients were observed. $7 / 8$ patients with food bolus impaction were previously diagnosed with esophageal stenosis. According to guidelines, 41 endoscopies (70\%) were warranted and $18(30 \%)$ were not. We compared our results from this period (III) with the two previous ones: before the adoption of guidelines (I) and the early period following the introduction of guidelines (II). The following was shown: endoscopy in $67 \%$ of patients with foreign body ingestion with $77 \%$ success rate (I), endoscopy in $20 \%$ of patients with $90 \%$ success rate, and in $34 \%$ of patients with $81 \%$ success rate (III).

Conclusion Global experiences suggest that endoscopic extraction is indicated in 10-20\% of cases of all foreign body ingestions in children. In the study period, in one third of $34 \%$ of patients in which the endoscopy was performed, it was not indicated according to current guidelines. Despite the existence of guidelines, tenacity and the vigilance of adherence to them decreases over time. Their existence by itself is not sufficient in reducing children's exposure to unnecessary and potentially harmful interventions.

\section{GASTROSCOPY IN PEDIATRICS - 4 YEAR EXPERIENCE}

Nedo Marčinković*, Matea Kovačić, Jurica Vuković, Duška Tješić Drinković, Irena SenečićČala, Lana Omerza, Mirna Natalija Aničic. Department of Pediatrics, University Hospital Center Zagreb, School of Medicine, University of Zagreb

10.1136/archdischild-2021-europaediatrics.282

Objective Comparing the referral diagnosis as an indication for EGD with final histological diagnosis, and to assess whether it was justified to perform these endoscopies in our patients.

Methods Retrospective analysis of patients who underwent gastroscopy with biopsy in the period from 1.1.2016. to 29.02.2020 at the Clinical Hospital Center Zagreb. The study did not include foreign body removal procedures, follow up endoscopies and endoscopies for the purpose of placing medical orthopedic aids. We have described the symptoms leading to referral for endoscopy, as well as their average duration prior to endoscopy. We analyzed the correlation between referral and final histological diagnosis.

Results In a cohort of a total of 100 patients, the most common indication for gastroscopy in 24/100 (24\%) was abdominal pain, and in approximately half of them $(13 / 24,54.2 \%)$ the histological cause of the discomfort was found (gastritis in 12 and celiac disease in 1 ). Of the 20 gastroscopies performed on suspicion of gastritis, in 12/20 (60\%) pathological substrate was found (9 gastritis, 2 eosinophilic esophagitis, 1 celiac disease). Due to celiac disease suspicion, we endoscopied 18 patients, of whom in $10(55.6 \%)$ celiac disease was histologically confirmed. In 6/11 (54.5\%) patients with dyspeptic symptoms diagnosis of gastritis was made after the endoscopy. In almost half of the patients, the pathohistological finding was normal. From 52 pathological findings; 31/52 (59.6\%) corresponds to gastritis, $12 / 52(23.1 \%)$ to celiac disease, $5 / 52$ (9.6\%) to eosinophilic esophagitis, and in 2 patients $(3.8 \%)$ esophageal varices and stomach polyps were found.

The average duration of discomfort was 10 months and 26 days, while the largest number of patients; 17 of them had symptoms for a year, 16 had problems for 6 months, 9 had symptoms for 2 years, and 8 had symptoms for 1 and 3 months, respectively before the endoscopy. It should be noted that 10 were asymptomatic and were referred for endoscopy on the basis of pathological laboratory findings (complete blood count, iron, antibodies to tissue transglutaminase) or specific anamnesis (body weight loss, failure to thrive). Endoscopy completion rate by entering into the distal end of duodenum was $100 \%$. We did not record any complications. 